\title{
Hybrid Methodology Based on Extension Neural Network for Fault Diagnosis of Photovoltaic Module
}

\author{
Shiue-Der Lu, ${ }^{1}$ Shao-En Wei, ${ }^{1}$ Meng-Hui Wang, ${ }^{*}$ \\ Hong-Wei Sian, ${ }^{2}$ and Cheng-Chien $\mathrm{Kuo}^{2}$ \\ ${ }^{1}$ Department of Electrical Engineering, National Chin-Yi University of Technology, \\ No. 57, Sec. 2, Zhongshan Rd., Taiping Dist., Taichung City 411030, Taiwan (R.O.C.) \\ ${ }^{2}$ Department of National Taiwan University of Science and Technology, \\ No. 43, Keelung Rd., Sec. 4, Da'an Dist., Taipei City 106335, Taiwan (R.O.C.)
}

(Received January 29, 2021; accepted April 16, 2021; online published 30 April, 2021)

Keywords: human-machine, fault diagnosis, extension neural network, chaotic synchronization detection method, photovoltaic (PV) module

We propose a human-machine graphic control fault diagnosis system based on an extension neural network (ENN) and the chaos synchronization detection method. A high-frequency signal is injected into a photovoltaic (PV) module to observe the voltage variation under different fault conditions, and the fault type is diagnosed using the proposed algorithm. Firstly, defects are introduced into the PV module and a high-frequency signal is injected by a signal generator. The high-frequency oscilloscope through the high-frequency sensor captures the voltage signal. The feature of the signal is calculated by the chaos synchronization detection method and a chaotic error scatter map is established. The chaos eye coordinates of the scatter diagram are used as eigenvalues for fault diagnosis. Finally, the ENN is used for fault diagnosis of the PV module. Also, from a comparison of analysis results with those of a traditional neural network, the ENN can identify the type of PV module fault rapidly and the recognition accuracy is as high as $87.5 \%$. Small changes in the voltage signal can be detected effectively by using the chaos synchronization detection method, and the preprocessing of big data is reduced. The PV module fault state is identified accurately to demonstrate the applicability of the method to PV module fault diagnosis.

\section{Introduction}

Rapid industrial development has led to an increased quality of life for people but has also led to a significant increase in the use of traditional fossil fuels. The world is confronted with the crises of fossil energy exhaustion and higher $\mathrm{CO}_{2}$ emissions, which contribute to the global greenhouse effect and climate change. To reduce these effects, various countries are looking for alternative energy sources. ${ }^{(1)}$ Multiple types of renewable energy can replace fossil energy, including hydropower, wind power, tidal power, and solar power. As renewable energy considers local climate conditions and the construction environment, solar power generation has low environmental pollution and produces no noise, employs a simple and modular structure, and

*Corresponding author: e-mail: wangmh@ncut.edu.tw https://doi.org/10.18494/SAM.2021.3307 
has high reliability. Therefore, solar power generation is undoubtedly a promising trend of renewable energy. Additionally, various countries have pushed energy policies actively in recent years, which has led to the rapid development of photovoltaic (PV) generation systems, which are becoming the main pillar of renewable energy development. ${ }^{(2-5)}$ With the construction and extensive use of PV generation systems, it is necessary to consider the aging of PV modules in the long-term operation of systems, which may be damaged in the outdoor environment, leading to reduced power generation and sometimes failure. Therefore, to avoid PV generation system faults, to reduce maintenance, labor, and cost, and to increase the efficiency of maintenance, PV generation system fault detection has become an important issue.

Some experts have performed research on the fault diagnosis of PV modules. Most studies used power generation data obtained from simulation software or by the actual measurement of the PV module of a PV generation system and used different algorithms to diagnose the fault in the PV generation system. In a previous study, MATLAB/Simulink software was used to build an equivalent mathematical model of a PV generation system to simulate fault diagnosis. ${ }^{(6)}$ However, nonlinear characteristics were exhibited as the atmospheric conditions changed, the simulation results were different from reality, and the parameter settings were difficult to calculate. In another study, maximum power point tracking (MPPT) was proposed on the basis of a thermal imager and PV controller, and a partial shadow fault was measured. ${ }^{(7)}$ While the majority of experiments on the aging and fault diagnosis for PV modules were conducted through thermal imaging, ${ }^{(8)}$ the application of thermal images has been limited due to high costs. An EL lens and drone were used to collect fault images to detect defects and faults effectively. ${ }^{(9)}$ However, it was necessary for a professional to review the fault images, the failure information could not be obtained immediately, and the EL lens and drone had a relatively high cost. Past studies used artificial neural networks for fault diagnosis. ${ }^{(10,11)}$ The diagnostic results were good, but to achieve more accurate recognition, a large amount of time and data were required for training and learning. A diagnostic method based on decision trees was proposed for a PV module. ${ }^{(12)}$ This method has a good recognition rate but the diagnostic process requires more than 1000 inter-comparisons and the experimental period is too long. A diagnostic method based on the Gisement solaire par télédetection: Solar Radiation by Teledectection (GISTEL) model was also proposed for improving the accuracy of the location of a PV cell from a satellite image obtained through fuzzy logic. ${ }^{(13)}$ However, this method is difficult to use in practice. Fault diagnosis by integrating chaos theory with extension theory could achieve high accuracy. ${ }^{(14)}$ However, the matter-element weight of extension theory must be adjusted through training, and the accuracy of diagnosis decreased significantly under some temperature and illuminance conditions.

In this study, we inject a high-frequency signal to capture the voltage drop of a PV module as a feature, which is combined with the Lorenz chaos synchronization detection method ${ }^{(15,16)}$ and an extension neural network $(\mathrm{ENN})^{(17)}$ to identify the fault types of a PV module. Type 1 is a surface rupture of the module, Type 2 is module aging, and Type 3 is a module bypass diode failure. Compared with the thermal image detection method and EL lens detection method, the proposed method does not require the costs of a thermal image lens and EL lens or a professional to operate a drone for patrol inspection. The proposed chaos synchronization detection method is highly sensitive to the input signal; if the input signal changes slightly, the output result 
presents a scale effect, which is favorable for extracting meaningful eigenvalues from the fault signal. Also, the ENN is a combination of extension theory and a neural network that provides a new correlation grade calculation for classification. It is characterized by parallel learning ability and a higher training iteration speed than that of a traditional neural network. It can perform the classification problem with range, continuous, and discrete outputs.

\section{PV Module Detection Method}

\subsection{Architecture of PV module fault diagnosis system}

This study uses an off-line detection mode, and three common PV module fault types are established: PV module breakage, PV module aging, and bypass diode failure of the PV module. Each combination comprises four units of a PV module connected in series to simulate fault conditions, and a high-frequency signal is injected into the PV module. The waveform is extracted using a high-frequency oscilloscope, the eigenvalues are extracted by the chaos synchronization detection method, and then the ENN is used for fault diagnosis. Figure 1 shows the architecture of the PV module fault diagnosis system and Fig. 2 shows the flow of the system.

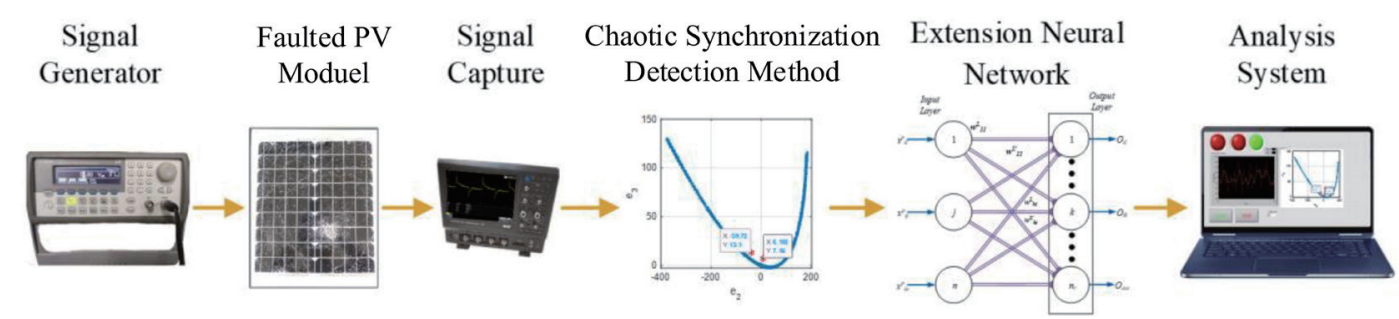

Fig. 1. (Color online) Architecture of PV module fault diagnosis system.

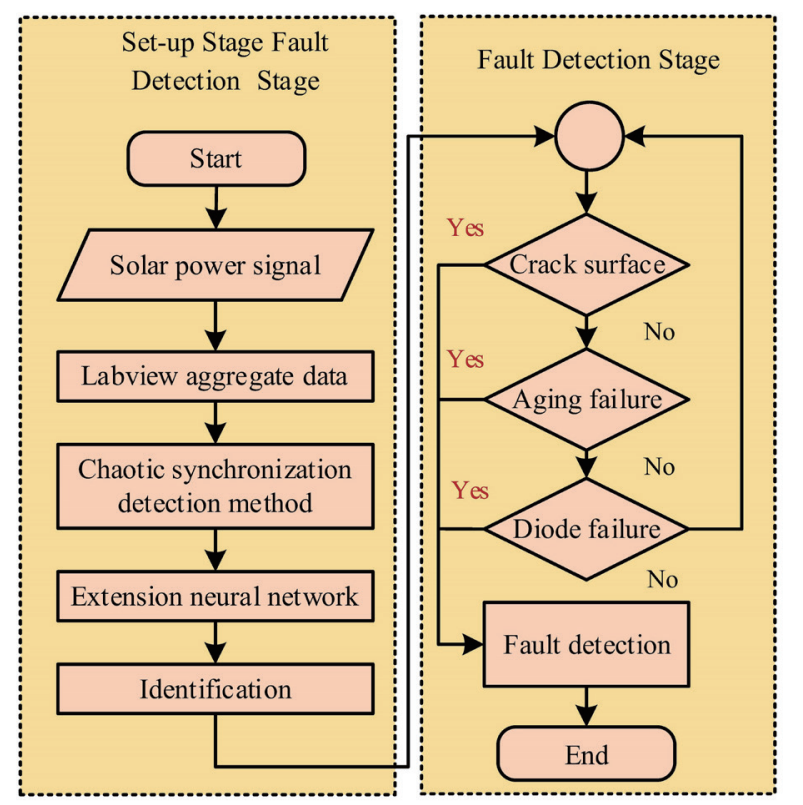

Fig. 2. (Color online) Flow of PV module fault diagnosis system. 


\subsection{PV module defect construction}

Long exposure to the sun ages PV modules, cracks are formed in PV modules due to external factors such as hail and trampling, ${ }^{(18)}$ or sometimes the bypass diode fails in PV modules. These factors can greatly reduce the power generating efficiency of the solar PV system. Figure 3 is a stereogram of a PV module defect. Related specifications are shown in Table 1. The construction method of different PV module fault types is described below.

\subsubsection{PV module breakage}

To produce a breakage in a PV module part of a PV module was placed under a blast burner until it reached a high temperature, and the physical phenomena of thermal expansion and contraction subsequently induced fracturing of the module surface, which imitated the hot spot effect on the heated surface. To simulate the module chip failure induced by human trampling or the strong impact of foreign matter, a normal PV module was knocked with a sharp object under an external force until a large area of reinforced glass was broken. Figure 4 shows the circuit used to measure the PV module. The PV was connected to a bypass diode in parallel and to an internal resistor (Ra). In this experiment, four PVs connected in series were employed. When a high-frequency square wave was injected by the signal generator, the fault signal waveform through the load was measured by an oscillograph.

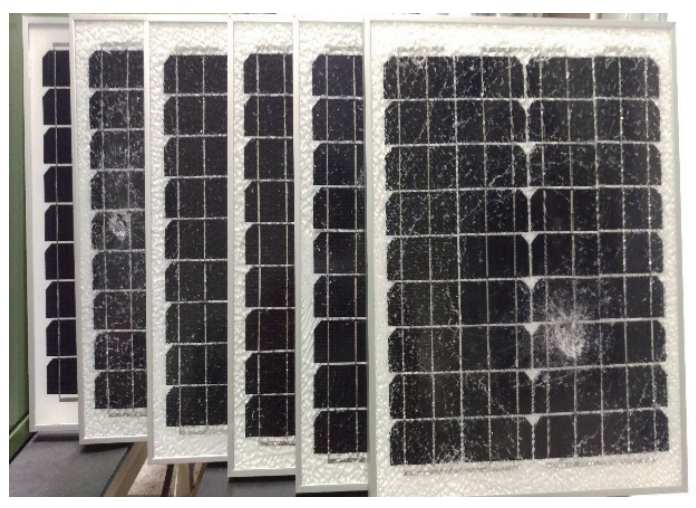

Table 1

PV module specifications

\begin{tabular}{lc}
\hline Project & Specification \\
\hline Max power $\left(P_{m}\right)$ & $20 \mathrm{~W}$ \\
Open circuit $\left(V_{o c}\right)$ & $22.4 \mathrm{~V}$ \\
Max power voltage $\left(V_{m p}\right)$ & $18.2 \mathrm{~V}$ \\
Max power current $\left(I_{m p}\right)$ & $1.10 \mathrm{~A}$ \\
Short circuit current $\left(I_{s c}\right)$ & $1.197 \mathrm{~A}$ \\
Mode dimensions $(L \times W \times H)$ & $426 \times 356 \times 25 \mathrm{~mm}^{3}$ \\
\hline
\end{tabular}

Fig. 3. (Color online) Stereogram of PV module defect.

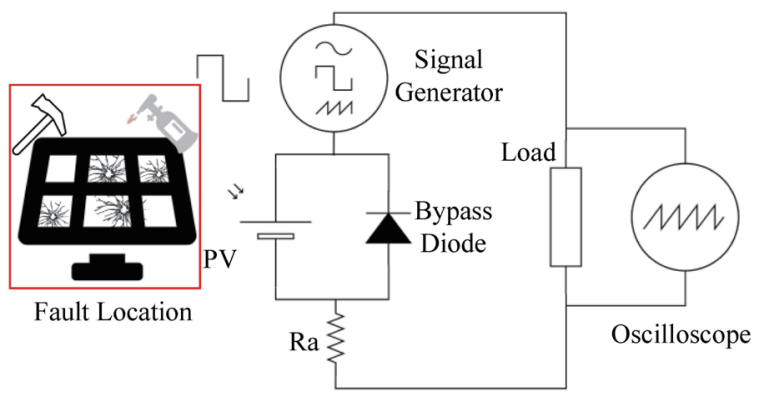

Fig. 4. (Color online) Circuit for measuring surface breakage of PV module. 


\subsubsection{PV module aging}

The elements of a PV module age with time or temperature changes. The aging of insulation will have a strong impact on the operational safety of electrical equipment. To construct an aged PV module using the aging fault construction method proposed in Ref. 19, additional resistors with resistances of 2 and $10 \Omega$ were separately applied to the PV module in series connection for testing. A variable resistor was connected to the PV module in series, i.e., the internal resistor (Ra) of the PV module in Fig. 4 was replaced by a variable resistor as shown in Fig. 5. The purpose was to detect the aging of the overall loop or regional lines and contact points. Similarly, four PV modules were connected in series to simulate a fault due to aging, a high-frequency square wave was imported by a signal generator, and the fault signal waveform through the load is measured by an oscillograph.

\subsubsection{PV module bypass diode failure}

In the PV conversion process, the PV module is likely to have problems due to external factors. For example, the shade of trees and bird droppings reduce electricity generation. A damaged or shielded region will generate heat, making a bypass diode very useful. This diode makes the current of normal PV modules pass by the faulty PV module to reduce the heating effect and protect the module against immediate overburn. The PV module bypass diode in Fig. 4 was removed to build the bypass diode failure state shown in Fig. 6, where four PV modules were similarly connected in series to simulate the aging fault. A high-frequency square wave was imported by a signal generator, and the fault signal waveform through the load was measured by an oscillograph.

\section{Proposed Fault Diagnosis Algorithm}

\subsection{Chaos synchronization detection method}

The American meteorologist Edward Norton Lorenz discussed the unstable characteristic of nonlinear systems and used a simple mathematical model of chaos theory to obtain nonperiodic

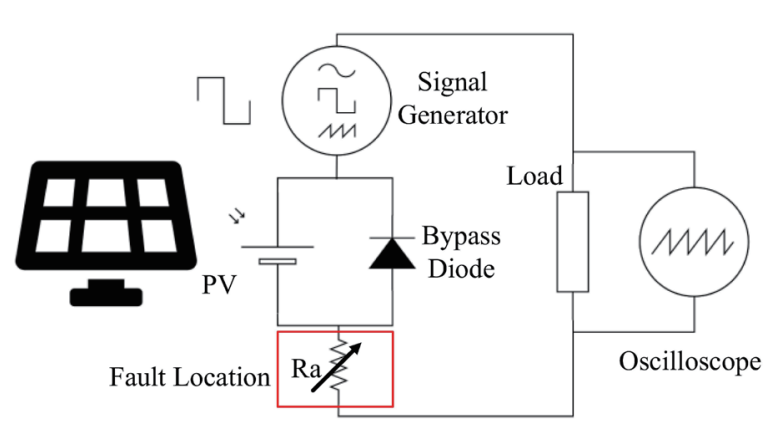

Fig. 5. (Color online) Measuring circuit for the aged PV module.

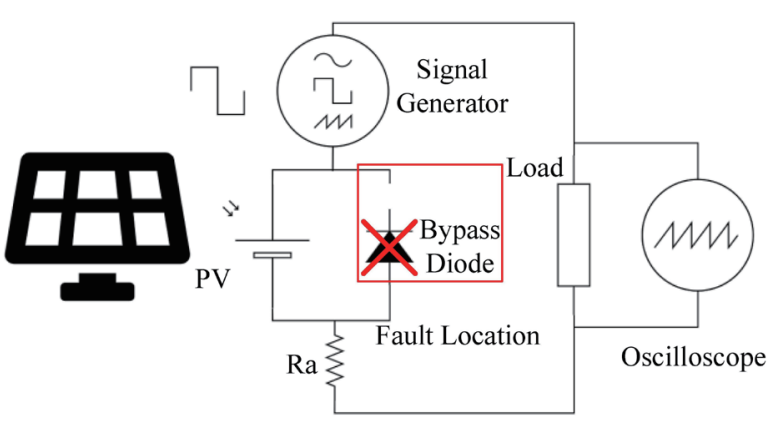

Fig. 6. (Color online) Measuring circuit for bypass diode failure of PV module. 
results. A signal generates chaotic attractors through chaos theory. The signal is orderly but has a nonperiodic motion trajectory. This trajectory produces a tremendous change from a small initial change because of the highly sensitive response to a microscale initial phenomenon, making chaos theory suitable for application to original data with a small variation range. Chaos theory is used in many fields such as biology, economics, physics, and engineering. Common chaos theories include the Lorenz chaos theory, Chen-Lee chaos theory, and Sprott chaos theory. Chaos theory has a master system (MS) and a slave system (SS), expressed as Eqs. (1) and (2), respectively. When the two systems receive different signals, the signal values are subtracted to generate the chaos dynamic error, and the MS and SS generate the motion trajectory of different dynamic errors for the engineering domain. A controller is added to the SS to track the MS. The MS and SS are gradually adjusted to the same motion trajectory by a controller, which is called chaotic synchronization action. ${ }^{(20)}$

Therefore, the captured PV module voltage signal is input in a master-slave synchronization system, and the dynamic errors of the natural chaos of the SS and MS are respectively subtracted by the master and slave chaotic systems to obtain the chaos dynamic error state.

$$
\begin{gathered}
S_{\text {master }}=\left\{\begin{array}{c}
\dot{x}_{1}=f_{1}\left(x_{1}, x_{2}, \ldots, x_{n}\right) \\
\dot{x}_{2}=f_{2}\left(x_{1}, x_{2}, \ldots, x_{n}\right) \\
\vdots \\
\dot{x}_{n}=f_{n}\left(x_{1}, x_{2}, \ldots, x_{n}\right)
\end{array}\right. \\
S_{\text {slave }}=\left\{\begin{array}{c}
\dot{y}_{1}=f_{1}\left(y_{1}, y_{2}, \ldots, y_{n}\right) \\
\dot{y}_{2}=f_{2}\left(y_{1}, y_{2}, \ldots, y_{n}\right) \\
\vdots \\
\dot{y}_{n}=f_{n}\left(y_{1}, y_{2}, \ldots, y_{n}\right)
\end{array}\right.
\end{gathered}
$$

Here, $f_{i}(i=1,2, \ldots, n)$ is a nonlinear function, Eq. (1) is subtracted from Eq. (2) to obtain the master-slave dynamic error, expressed as Eq. (3), and the calculated dynamic error equation is expressed as Eq. (4).

$$
\begin{gathered}
\left\{\begin{array}{c}
e_{1}=y_{1}-x_{1} \\
e_{2}=y_{2}-x_{2} \\
\vdots \\
e_{n}=y_{n}-x_{n}
\end{array}\right. \\
\left\{\begin{array}{c}
\dot{e}_{1}=f_{1}\left(y_{1}, y_{2}, \ldots, y_{n}\right)-f_{1}\left(x_{1}, x_{2}, \ldots, x_{n}\right) \\
\dot{e}_{2}=f_{2}\left(y_{1}, y_{2}, \ldots, y_{n}\right)-f_{2}\left(x_{1}, x_{2}, \ldots, x_{n}\right) \\
\vdots \\
\dot{e}_{n}=f_{n}\left(y_{1}, y_{2}, \ldots, y_{n}\right)-f_{n}\left(x_{1}, x_{2}, \ldots, x_{n}\right)
\end{array}\right.
\end{gathered}
$$


The Lorenz master and slave chaos systems are mathematical models established on the basis of the chaos theory and can generate infinite models. In this study we use the Lorenz equation and two Lorenz chaos systems, i.e., the Lorenz master and slave chaos systems expressed as Eqs. (5) and (6), respectively.

$$
\begin{gathered}
L_{\text {master }}=\left\{\begin{array}{l}
\dot{x}_{1}=\alpha\left(x_{2}-x_{1}\right) \\
\dot{x}_{2}=\beta x_{1}-x_{1} x_{3}-x_{2} \\
\dot{x}_{3}=x_{1} x_{2}-\gamma x_{3}
\end{array}\right. \\
L_{\text {slave }}=\left\{\begin{array}{l}
\dot{y}_{1}=\alpha\left(y_{2}-y_{1}\right) \\
\dot{y}_{2}=\beta y_{1}-y_{1} y_{3}-y_{2} \\
\dot{y}_{3}=y_{1} y_{2}-\gamma y_{3}
\end{array}\right.
\end{gathered}
$$

The chaos dynamic error is defined as $e_{1}=y_{1}-x_{1}, e_{2}=y_{2}-x_{2}$, and $e_{3}=y_{3}-x_{3}$, the dynamic error vector is $\boldsymbol{e}=\left[e_{1}, e_{2}, e_{3}\right]^{T}$, and the dynamic error equation of the Lorenz master and slave chaos systems is represented in a matrix as follows: ${ }^{(21)}$

$$
\left[\begin{array}{l}
\dot{e}_{1} \\
\dot{e}_{2} \\
\dot{e}_{3}
\end{array}\right]=\left[\begin{array}{ccc}
-\alpha & \alpha & 0 \\
\beta & -1 & 0 \\
0 & 0 & -\gamma
\end{array}\right]\left[\begin{array}{l}
e_{1} \\
e_{2} \\
e_{3}
\end{array}\right]+\left[\begin{array}{c}
0 \\
-e_{1} e_{3} \\
e_{1} e_{2}
\end{array}\right]
$$

$x$ is set as the MS and its initial value is zero. $y$ is set as the SS with the PV voltage as its initial value. $\alpha, \beta$, and $\gamma$ are coefficients for adjusting the error $(\alpha=10, \beta=28, \gamma=8 / 3)$, and the Lorenz master and slave chaos systems are derived from this group of error coefficients. ${ }^{(22)}$ The distance of chaos eye coordinates in the chaotic scatter diagram is calculated by using the largest rootmean-square deviation. The chaos eye coordinate distances $Z$ of chaotic scatter diagrams $e_{1} e_{2}$, $e_{2} e_{3}$, and $e_{1} e_{3}$ are calculated using Eq. (8). The values are compared to obtain the maximum value, defined as the preferred eigenvalue. Table 2 shows the longest distance between two chaos eye coordinate distances from the $e_{2} e_{3}$ chaotic scatter diagram, and the chaos eye coordinate value is used as the eigenvalue of the PV module diagnosis system. The chaos dynamic error scatter map is defined as shown in Fig. 7.

$$
Z=\sqrt{\left(X_{11}-X_{21}^{\prime}\right)^{2}+\left(Y_{11}-Y_{21}^{\prime}\right)^{2}+\left(X_{12}-X_{22}^{\prime}\right)^{2}+\left(Y_{12}-Y_{22}^{\prime}\right)^{2}}
$$

Table 2

Comparison of PV module fault feature values.

\begin{tabular}{lccc}
\hline Chaotic system & $e_{1} e_{2}$ & $e_{2} e_{3}$ & $e_{1} e_{3}$ \\
\hline Chaos eye coordinate distance $(Z)$ & 14.99 & 40.36 & 12.55 \\
\hline
\end{tabular}




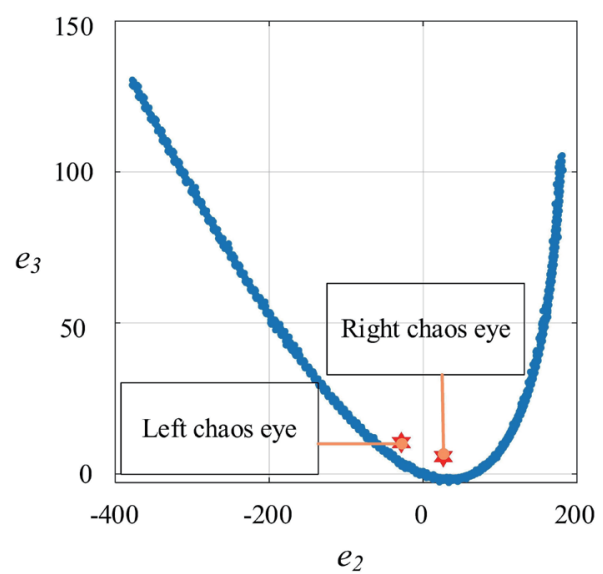

Fig. 7. (Color online) Scatter map of chaos dynamic error.

\subsection{Extension neural network}

Extension theory provides a new correlation grade calculation for classification processing, and the predominant feature of a neural network is parallel learning ability. Therefore, their combination provides a new classification recognition method. The ENN can perform supervised learning and possesses the ability to solve classification problems with consecutive inputs and discrete outputs. ${ }^{(17)}$

Figure 8 shows the basic structure of an ENN that includes an input layer and an output layer. A characteristic sample is obtained at the node of the input layer, and a preset weighting parameter is used to generate an input pattern image. In this network architecture, each connected input node and output node has two weights. One weight represents the minimum value of the input feature of this node, and the other weight represents the maximum value of the input of the node. For example, $W_{k j}^{U}$ and $W_{k j}^{L}$ are connected at the $j$ th input node and $k$ th output node. A flow chart of the ENN is shown in Fig. 9.

\subsubsection{Extension neural network learning rule}

The ENN is used for supervised learning, and an output value identical to the target is achieved by adjusting the weight or reducing the error of the output value. ${ }^{(23)}$ Several variables are defined before learning. Let the learning sample be $X=\left\{x_{1}, x_{2}, \ldots, x_{N P}\right\}$ and $N_{P}$ be the number of training samples. The $i$ th sample is $X_{i}^{p}=\left\{x_{i 1}^{P}, x_{i 2}^{P}, \ldots, x_{i n c}^{P}\right\}$, the total number of characteristic samples is $n_{c}$, and the type of the $i$ th sample is $p$. To evaluate the correctness of ENN prediction, the testing error is set as $N_{m}$ and the total error ratio is set as

$$
E_{T}=\frac{N_{m}}{N_{p}}
$$




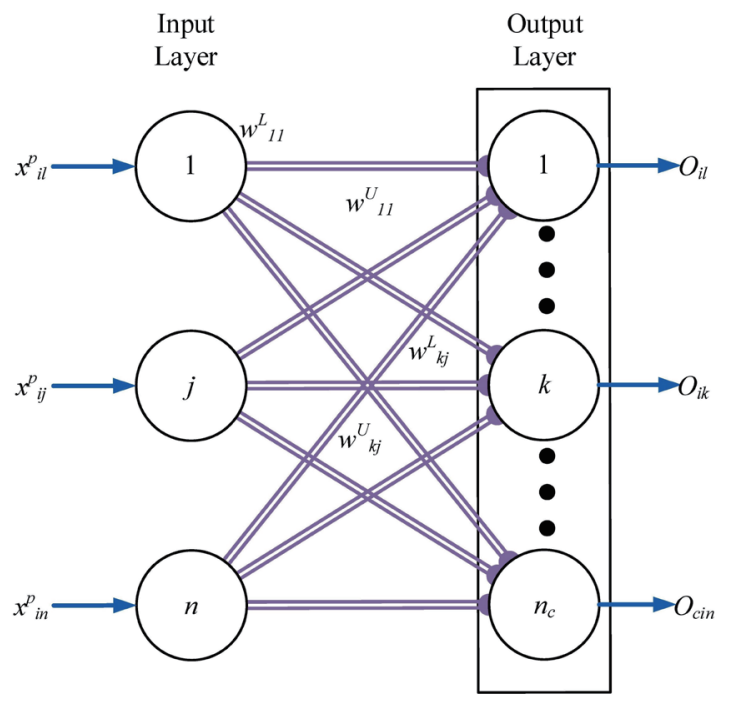

Fig. 8. (Color online) Basic structure of ENN.

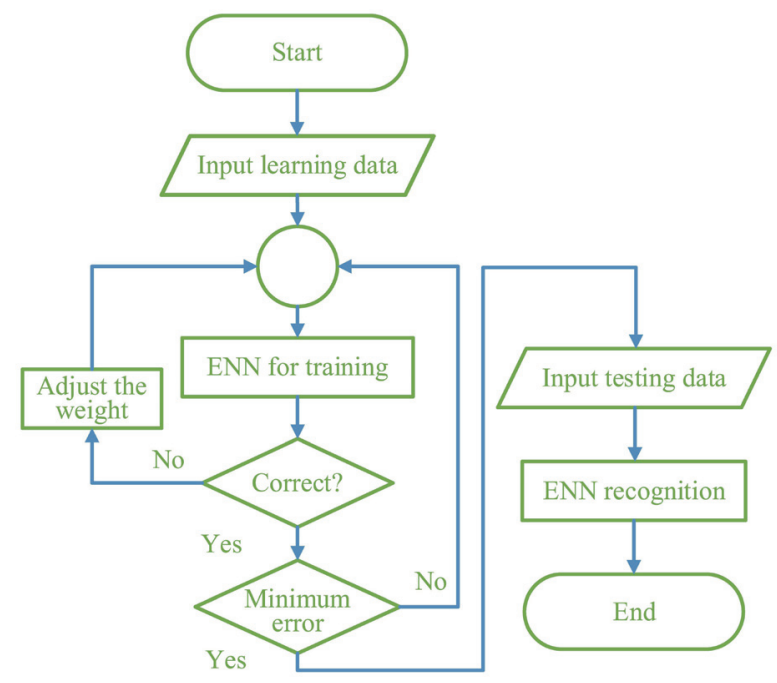

Fig. 9. (Color online) ENN flow chart.

The ENN learning rule is described below:

Step 1: Set the weights between an input node and an output node as Eqs. (10) and (11) in accordance with the characteristic model.

$$
\begin{gathered}
w_{k j}^{U}=\max _{i \in N_{P}}\left\{x_{i j}^{k}\right\} \\
w_{k j}^{L}=\min _{i \in P_{N}}\left\{x_{i j}^{k}\right\}
\end{gathered}
$$

Step 2: Calculate the median weight of each feature, expressed as Eqs. (12) and (13).

$$
\begin{gathered}
Z_{k}=\left\{Z_{k 1}, Z_{k 2}, \ldots, Z_{k n}\right\} \\
Z_{k j}=\left(W_{k j}^{L}+W_{k j}^{U}\right) / 2 \text { for } k=1,2, \ldots, n_{c} ; j=1,2, \ldots, n_{c}
\end{gathered}
$$

Step 3: Read the ith training sample and characteristic number $p$, expressed as Eq. (14).

$$
X_{i}^{p}=\left\{X_{i 1}^{p}, X_{i 2}^{p}, \ldots, X_{i n c}^{p}\right\}, P \in n_{c}
$$

Step 4: Use the extension distance to calculate the distance from the $k$ th cluster to the sample, expressed as Eq. (15). 


$$
E D_{i k}=\sum_{j=1}^{n}\left[\frac{\left|x_{i j}^{p}-z_{k j}\right|-\frac{\left(w_{k j}^{U}-w_{k j}^{L}\right)}{2}}{\left|\frac{\left(w_{k j}^{L}-w_{k j}^{U}\right) \mid}{2}\right|}+1\right] \text { for } k=1,2, \ldots, n_{c}
$$

Step 5: Look for $k^{*}, E D_{i k^{*}}=\min \left\{E D_{i k^{*}}\right\}$. If $k^{*}=p$, proceed to Step 7; otherwise, proceed to Step 6.

Step 6: The new $p$ th and $k^{*}$ th weights are described below.

The $p$ th and $k^{*}$ th weight medians are updated using Eqs. (16) and (17), respectively.

$$
\begin{aligned}
& Z_{p j}^{n e w}=Z_{p j}^{\text {old }}+\left(X_{i j}^{p}-Z_{p j}^{\text {old }}\right), P \in n_{c} \\
& Z_{k^{*} j}^{n e w}=Z_{k^{*} j}^{\text {old }}+\left(X_{i j}^{p}-Z_{k^{*} j}^{\text {old }}\right), P \in n_{c}
\end{aligned}
$$

The $p$ th and $k^{*}$ th weights are updated using Eqs. (18)-(21), wherein $\eta$ is the learning rate.

$$
\begin{aligned}
& W_{p j}^{L(\text { new })}=W_{p j}^{L(\text { old })}+\eta\left(X_{i j}^{p}-Z_{p j}^{\text {old }}\right) \\
& W_{p j}^{L(\text { new })}=W_{p j}^{U(\text { old })}+\eta\left(X_{i j}^{p}-Z_{p j}^{\text {old }}\right) \\
& W_{p j}^{L(\text { new })}=W_{k^{*} j}^{L(\text { old })}+\eta\left(X_{i j}^{p}-Z_{k^{*} j}^{\text {old }}\right) \\
& W_{p j}^{L(\text { new })}=W_{k^{*} j}^{U(\text { old })}+\eta\left(X_{i j}^{p}-Z_{k^{*} j}^{\text {old }}\right)
\end{aligned}
$$

Step 7: Repeat Steps 3 to 6 until all samples have been classified.

Step 8: If the classification has converged or the total error rate reaches a minimum standard value, stop the process; otherwise, return to Step 3.

\subsubsection{Extension neural network recognition stage}

After the ENN has completed learning, classification or recognition can be performed using the following algorithm.

Step 1: Read the weighting matrix learned by the ENN.

Step 2: Calculate the median of each center, expressed as Eqs. (12) and (13). 
Step 3 Read the test sample expressed as

$$
X_{t}=\left\{X_{t 1}, X_{t 2}, \ldots, X_{m}\right\}
$$

Step 4: Use the proposed extension distance to calculate the distance from each cluster.

Step 5: Look for $k^{*}$ using the cluster types of $E D_{i k^{*}}=\min \left\{E D_{i k}\right\}$ and $O_{i k^{*}}=1$.

Step 6: If all test samples are classified, stop the process; otherwise, return to Step 3.

\section{Actual Measurement and Analysis of Results}

\subsection{PV module signal measurement and extraction}

Figure 10 shows the PV module signal testing platform for this study; the PV module is measured in an off-line mode without a power supply. Four $20 \mathrm{~W}$ PV modules are connected in series to form a loop, and the PV module is turned over to avoid the PV effect. The waveform generator generates a $250 \mathrm{kHz}$ square wave signal, which is injected into the test PV module output end. The high-frequency oscilloscope captures the variations of the voltage waveform of inductive loads connected to the loop in series.

A WAVESURFER 3054 oscillograph is used in this study. The upper bound of the bandwidth is $500 \mathrm{MHz}$, the sampling rate is $4 \mathrm{GS} / \mathrm{s}$, the input is four analog channels, the maximum withstand voltage is $400 \mathrm{~V}$, the probe has a magnification of 1 , the withstand voltage is $150 \mathrm{~V}$, and the effective extraction frequency is $100 \mathrm{MHz}$.

Three types of PV module faults are constructed in this study. These faults are breakage, aging, and bypass diode failure. Two complete cycles are extracted from each fault type as data samples. The data sampling time is $8 \mu \mathrm{s}$, the sampling frequency is $4 \mathrm{GS} / \mathrm{s}$, and there are 32000 sampling points. The measured waveforms of PV module breakage, aging, and bypass diode failure are respectively shown in Figs. 11-13.

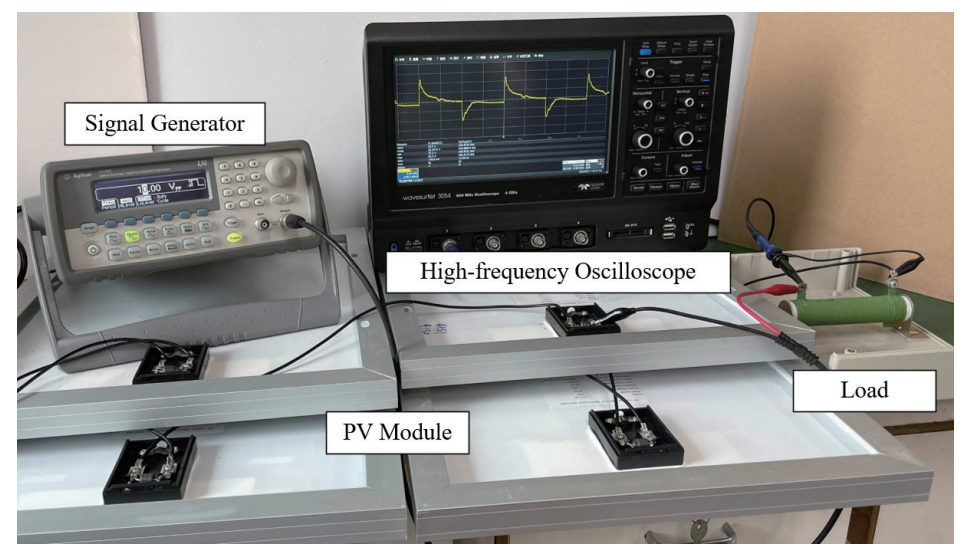

Fig. 10. (Color online) PV module signal testing platform. 


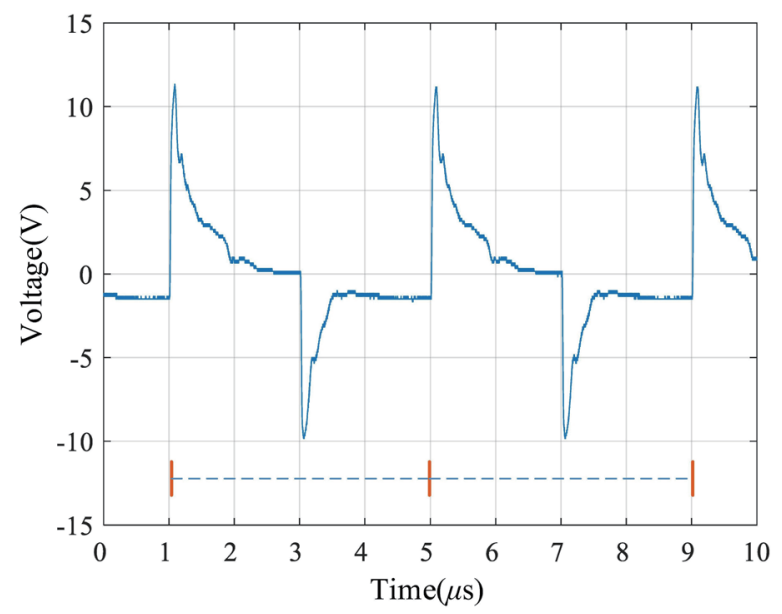

Fig. 11. (Color online) Waveform of PV module breakage.

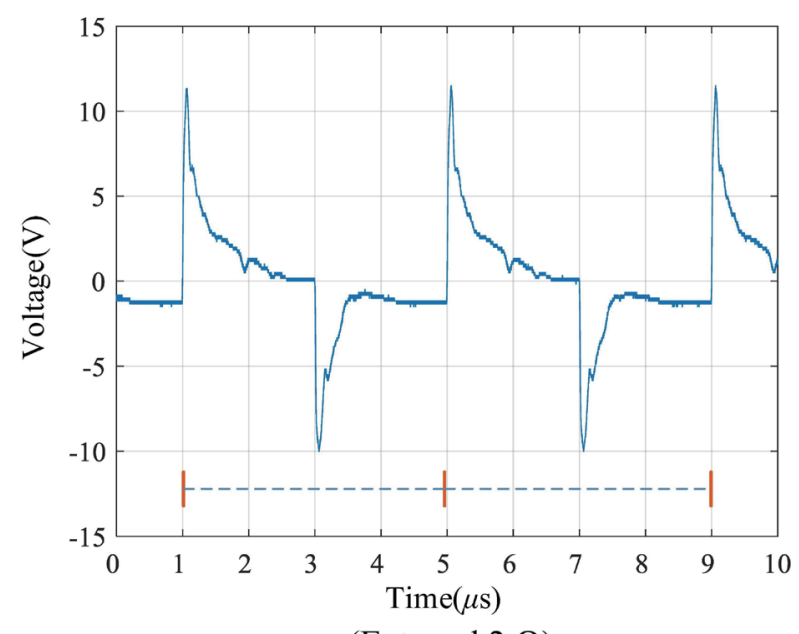

(External $2 \Omega$ )

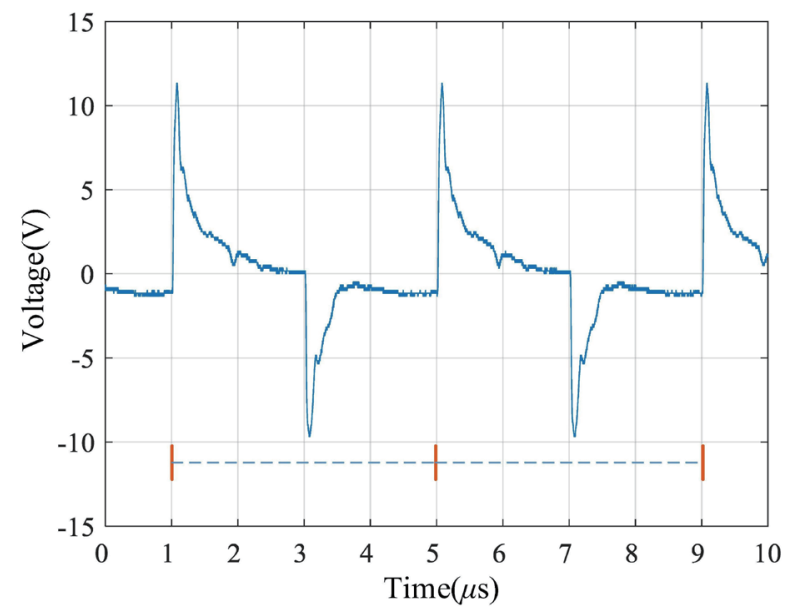

$($ External $10 \Omega$ )

Fig. 12. (Color online) Waveform of PV module aging.

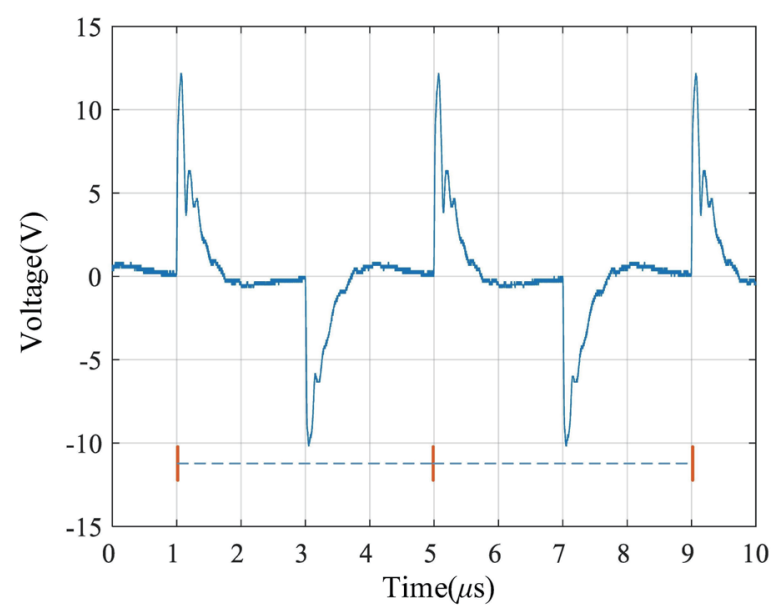

Fig. 13. (Color online) Waveform of PV module bypass diode failure. 


\subsection{Extraction of signal characteristic value by chaos synchronization detection method}

There are 32000 measured voltage signals for each type of PV module fault. It is impossible to obtain explicit features from the waveform. Therefore, we use the chaos synchronization detection method to calculate the 32000 voltage data. The chaos eye coordinates of the chaotic scatter diagram are extracted as the eigenvalues for fault recognition. This method effectively compresses mass data and extracts representative eigenvalues. The accuracy of ENN recognition is thus enhanced effectively.

The three voltage waveforms of the PV module are calculated by the Lorenz chaos synchronization detection method. The chaotic scatter diagram and chaos eye coordinates are shown in Figs. 14-16. The chaos eye coordinates of the PV module fault types are represented as chaos eye coordinate distributions, as shown in Fig. 17, which provide the eigenvalues for ENN recognition and fault classification.

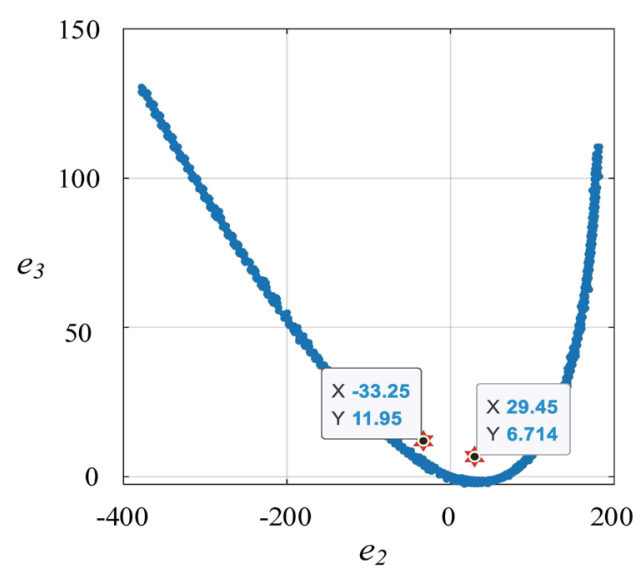

Fig. 14. (Color online) Chaotic scatter diagram and chaos eye coordinates of PV surface rupture.

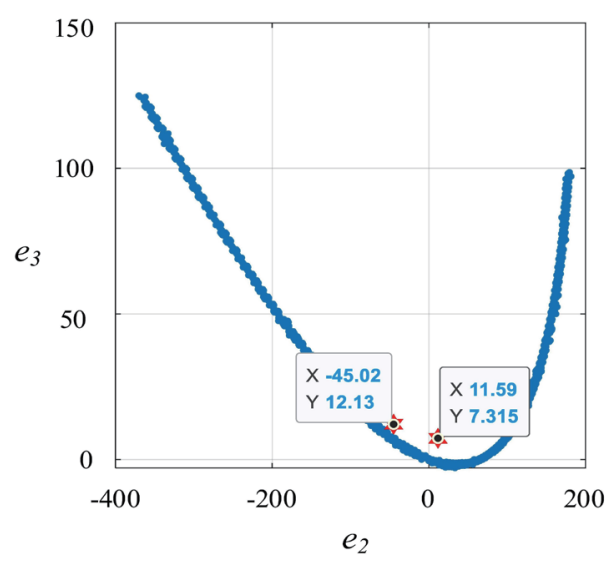

Fig. 15. (Color online) Chaotic scatter diagram and chaos eye coordinates of PV module aging.

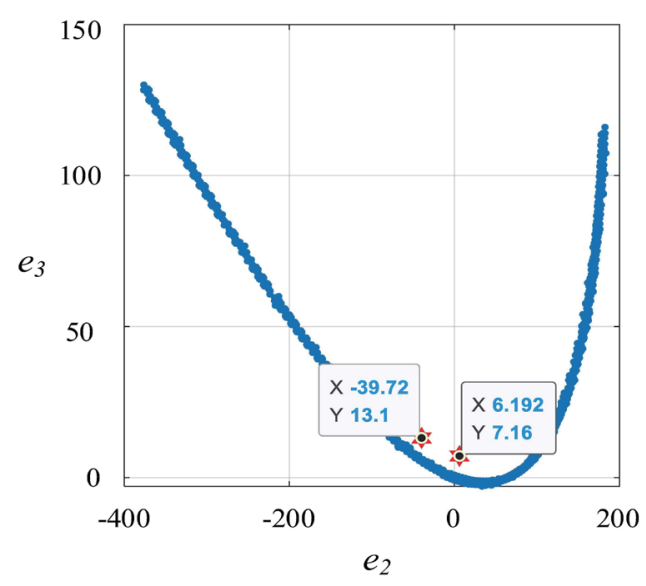

Fig. 16. (Color online) Chaotic scatter diagram and chaos eye coordinates of PV module bypass diode failure. 


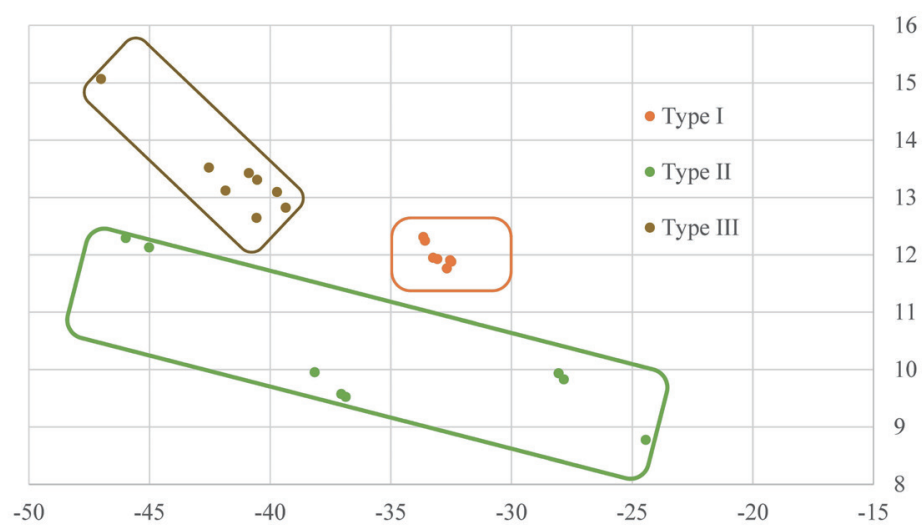

Fig. 17. (Color online) Chaos eye coordinates of PV module fault types represented as chaos eye coordinate distributions.

\subsection{ENN recognition result and comparison}

We use MATLAB to compose the ENN to implement the system for recognizing PV module faults. Figure 18 shows the ENN training curve. It can be observed that the error rate is zero when the training is iterated nine times, meaning that the ENN has completed its training and learns successfully. The recognition accuracy rate is $87.5 \%$.

The chaos eye eigenvalue for PV module fault types was employed for training and recognition using the most commonly adopted backpropagation network (BPN), and the results were compared with those from the ENN. As shown in Table 3, the recognition results of the ENN yield the highest accuracy rate of $87.5 \%$, followed by the $75 \%$ for probabilistic neural network (PNN). Moreover, the accuracy rate from the recognition results of the ENN is superior to the rates from all other traditional neural network algorithms with the largest difference being $45 \%$.

\subsection{ENN recognition result and comparison}

LabVIEW developed by National Instruments has a special link library for hardware, instrument control, and analytical software with powerful functions and high applicability. We used LabVIEW graphic control program software to implement the diagnostic recognition system of human-machine interface graphic control for the PV module, which is convenient for maintenance and operating staff to perform fault diagnosis. Figure 19 shows the humanmachine graphic control detection interface developed for the PV model. The PV module voltage signal is transferred to the human-machine interface system, then chaos synchronization detection and ENN recognition of the PV module are performed instantly and the fault diagnosis result is displayed.

Figure 19 shows the fault diagnosis output result of PV module breakage. The humanmachine interface has the following functions.

(a) Fault display light signal: the diagnostic result after ENN recognition of the feature value.

(b) Voltage waveform: the measured signal of the PV module. 


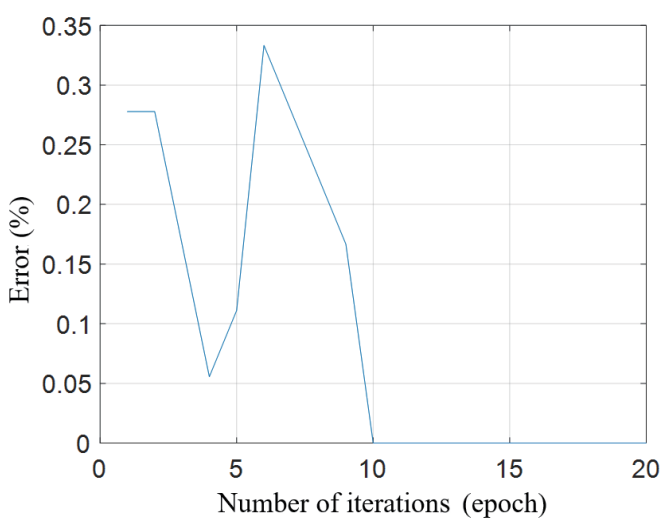

Fig. 18. (Color online) ENN training curve.

Table 3

Recognition results of ENN and other algorithms.

\begin{tabular}{lrccc}
\hline Algorithm & Epoch & Learning rate (\%) & Accuracy rate (\%) & Ranking \\
\hline ENN & 20 & 100 & 87.5 & 1 \\
PNN & 1 & N/A & 75 & 3 \\
MNN (4-9-3) & 1000 & 82.1 & 62.5 & 2 \\
MNN (4-18-3) & 1000 & 81.2 & 50 & 4 \\
MNN (4-10-3) & 1000 & 67.9 & 47.5 & 5 \\
MNN (4-17-3) & 1000 & 60.3 & 42.5 & 6 \\
\hline
\end{tabular}

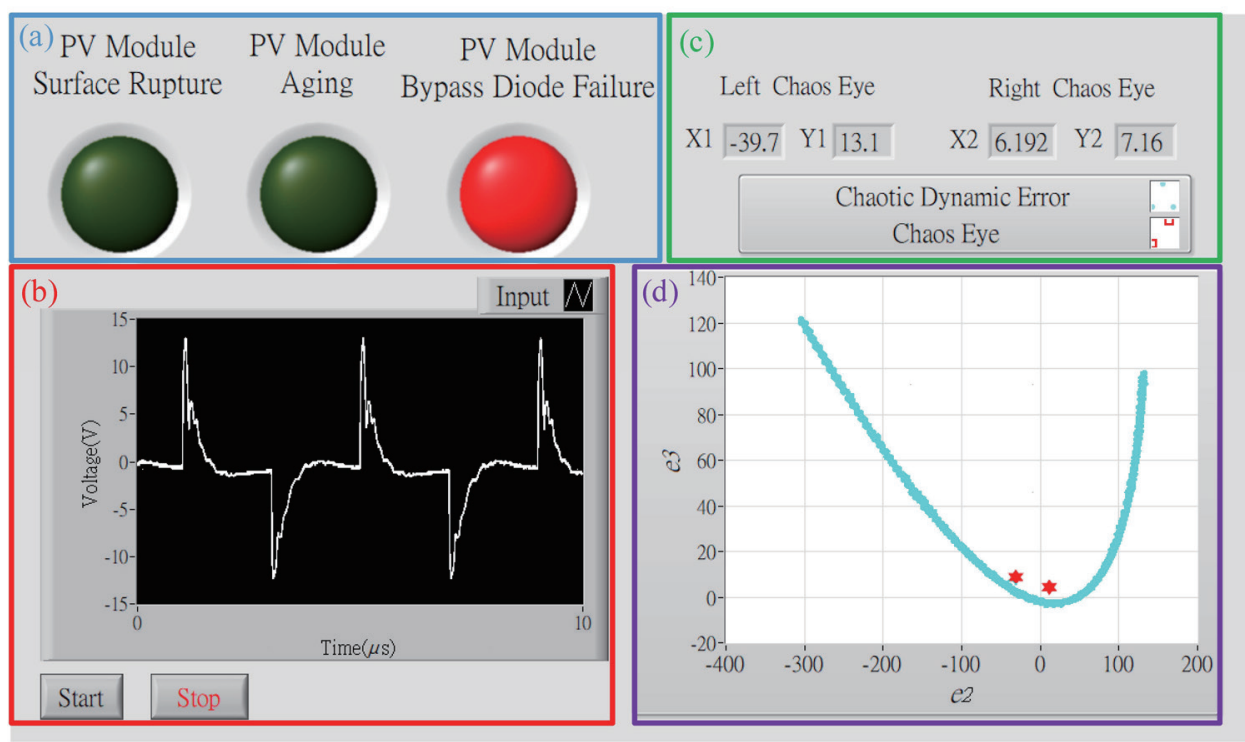

Fig. 19. (Color online) Human-machine interface graphic control diagnostic recognition system.

(c) Chaos eye coordinate value: the chaos eye coordinate value of the chaos scatter diagram is the feature value of the PV module voltage.

(d) Chaos scatter diagram: the chaos scatter diagram after theoretical calculation of the PV module voltage signal by chaotic synchronization detection. 


\section{Conclusions}

We proposed a method for PV module fault recognition based on the ENN and chaos synchronization feature extraction. Three common fault types were constructed for the PV module. The chaos eye coordinates of the Lorenz chaos synchronization system were used as the feature of fault diagnosis. The advantage of this method is that it compresses a large amount of data while effectively extracting eigenvalues from this data. The trained ENN was used for identifying fault types. The recognition accuracy rate of the proposed method is as high as $87.5 \%$, superior to that of a traditional neural network, proving that the method proposed in this study is effective for PV module fault diagnosis. Additionally, we used LabVIEW to develop a human-machine interface graphic control diagnostic program, which is integrated with a chaos synchronization detection method and the ENN to build a real-time measurement and recognition system. It is implemented in the PV module fault diagnosis recognition system.

\section{Acknowledgments}

The authors gratefully acknowledge the financial support of the Ministry of Science and Technology of Taiwan, under contract numbers: MOST 109-2221-E-167-009, MOST 108-2622-E-167-015-CC3, and MOST 108-2221-E-167-018-MY2.

\section{References}

1 F. D. Kanellos, E. Grigoroudis, C. Hope, V. S. Kouikoglou, and Y. A. Phillis: IEEE Syst. J. 11 (2017) 4. https:// doi.org/10.1109/JSYST.2014.2376493

2 R. M. Elavarasan, G.M. Shafiullah, S. Padmanaban, N. M. Kumar, A. Annam, A. M. Vetrichelvan, L. MihetPopa, and J. B. Holm-Nielsen: IEEE Access 8 (2020) 74432. https://doi.org/10.1109/ACCESS.2020.2988011

3 O. Ogunrinde, E. Shittu, and K. K. Dhanda: IEEE Eng. Manage. Rev. 46 (2018) 4. https://doi.org/10.1109/ EMR.2018.2880445

4 G. P. Holdmann, R. W. Wies, and J. B. Vandermeer: Proc. IEEE 107 (2019) 9. https://doi.org/10.1109/ JPROC.2019.2932755

5 Taipei times: https://www.taipeitimes.com/News/front/archives/2020/10/15/2003745182 (accessed October 2020).

6 A. Q. Al-Shetwi and M. Z. Sujod: Proc. 2016 Int. Conf. Advances in Electrical, Electronic and Systems Engineering (IEEE, 2016) 488-494. https://doi.org/10.1109/ICAEES.2016.7888094

7 Y. Hu, W. Cao, J. Wu, B. Ji, and D. Holliday: IEEE Trans. Power Electron. 29 (2014) 11. https://doi.org/10.1109/ TPEL.2014.2325062

8 Z. Zou, Y. Hu, B. Gao, W. L. Woo, and X. Zhao: J. Appl. Phys. 115 (2014) 4. https://doi.org/10.1063/1.4863783

9 G. Alves dos Reis Benatto, C. Mantel, S. Spataru, A. A. S. Lancia, N. Riedel, S. Thorsteinsson, P. B. Poulsen, H. Parikh, S. Forchhammer, and D. Sera: IEEE J. Photovoltaics 10 (2020) 3. https://doi.org/10.1109/ JPHOTOV.2020.2978068

10 S. Syafaruddin, E. Karatepe, and T. Hiyama: Proc. 2011 16th Int. Conf. Intelligent System Applications to Power Systems (IEEE, 2011) 1-6. https://doi.org/10.1109/ISAP.2011.6082219

11 S. Laamami, M. Benhamed, and L. Sbita: Proc. 2017 Int. Conf. Green Energy Conversion Systems (IEEE, 2017) 1-7. https://doi.org/10.1109/GECS.2017.8066211

12 Y. Zhao, L. Yang, B. Lehman, J. de Palma, J. Mosesian, and R. Lyons: Proc. 2012 27th Annu. IEEE Applied Power Electronics Conf. Exposit. (IEEE, 2012) 93-99. https://doi.org/10.1109/APEC.2012.6165803

13 M. Tadj, K. Benmouiza, A. Cheknane, and S. Silvestre: Energy Convers. Manage. 80 (2014) 298. https://doi. org/10.1016/j.enconman.2014.01.030

14 C. T. Hsieh, H. T. Yau, and J. Shiu: Int. J. Photoenergy 2014 (2014) 9. https://doi.org/10.1155/2014/759819 
15 A. Faqih, B. Kamanditya, and B. Kusumoputro: Proc. 2018 Int. Conf. Computer, Information and Telecommunication Systems (IEEE, 2018) 1-5. https://doi.org/10.1109/CITS.2018.8440187

16 B. Li, P. Li, L. Zhang, and M. Ma: Proc. 2008 China Int. Conf. Electricity Distribution (IEEE, 2008) 1-5. https://doi.org/10.1109/CICED.2008.5211666

17 M. H. Wang and C. P. Hung: Proc. Int. Joint Conf. Neural Networks 1 (2003) 399-403. https://doi.org/10.1109/ IJCNN.2003.1223379

18 A. Haque, K. V. S. Bharath, M. A. Khan, I. Khan, and Z. A. Jaffery: Energy Sci. Eng. 7 (2019) 3. https://doi. org/10.1002/ese 3.255

19 J. Huang, R. Wai, and W. Gao: IEEE Access 7 (2019) 70919. https://doi.org/10.1109/ACCESS.2019.2919337

20 M. A. Khelifa, A. Boukabou, and N. Hammami: Proc. 2013 Int. Conf. Computer Applications Technology (IEEE, 2013) 1-2. https://doi.org/10.1109/ICCAT.2013.6521983

21 C. H. Huang, C. H. Lin, and C. L. Kuo: IEEE Trans. Power Delivery 26 (2010) 2. https://doi.org/10.1109/ TPWRD.2010.2090176

22 H. T. Yau and M. H. Wang: IET Renewable Power Gener. 9 (2015) 6. https://doi.org/10.1049/iet-rpg.2014.0269

23 K. H. Chao, M. H. Wang, and Y. H. Lee: Proc. 2011 Int. Conf. Machine Learning and Cybernetics (IEEE, 2011) 654-660. https://doi.org/10.1109/ICMLC.2011.6016761 